
\title{
28 Research Suare \\ Efficacy and Safety of Two Salts of Trientine in the Treatment of Wilson Disease
}

\section{France Woimant}

Hopital Lariboisiere Service de Neurologie

\section{Dominique Debray}

Hopital Necker-Enfants Malades: Hopital universitaire Necker-Enfants malades

\section{Erwan Morvan}

Fondation Ophtalmologique Adolphe de Rothschild: La Fondation Adolphe de Rothschild

\section{Mickael Alexandre Obadia ( $\nabla$ mickael.alexandre.obadia@gmail.com )}

The Fondation Adolphe de Rothschild Hospital: La Fondation Adolphe de Rothschild https://orcid.org/0000-0002-4221-6163

\section{Aurélia Poujois}

Fondation Ophtalmologique Adolphe de Rothschild: La Fondation Adolphe de Rothschild

\section{Research}

Keywords: Wilson disease, medication adherence, chronic disease, Trientine salts, D-Penicillamine, zinc salts, efficacy, safety

Posted Date: May 24th, 2021

DOI: https://doi.org/10.21203/rs.3.rs-505524/v1

License: (c) (i) This work is licensed under a Creative Commons Attribution 4.0 International License.

Read Full License 
Efficacy and safety of two salts of trientine in the treatment of Wilson disease

France Woimant ${ }^{1,2}$, Dominique Debray ${ }^{1,3}$, Erwan Morvan ${ }^{1,4}$, Mickael Alexandre Obadia ${ }^{1,4}$, Aurélia Poujois ${ }^{1,4}$

\section{Affiliations}

${ }^{1}$ National Reference Centre for Wilson's Disease, Rothschild Foundation Hospital, Paris, France

${ }^{2}$ Department of Neurology, Lariboisière University Hospital, AP-HP, Paris, France

${ }^{3}$ Department of liver Pediatrics, Necker University Hospital, AP-HP, Paris, France

${ }^{4}$ Department of Neurology, Rothschild Foundation Hospital, Paris, France

\section{Correspondence}

Dr Mickael Alexandre Obadia, National Reference Centre for Wilson's Disease Neurology Department, Rothschild Foundation Hospital, 29 rue Manin, 75019 Paris, France mickael.alexandre.obadia@gmail.com

Word counts for the text: 2803

\section{Number of tables: 4}

\section{Number of Figures: 1}

\section{Author contributions:}

Designing and conceptualization of research studies: FW, AP

Conducting experiments: FW, DD, AP

Analyzing data: FW, DD, EM, MAO, AP

Writing the first draft of the manuscript: FW, AP

Critical revisions of the manuscript: DD, EM, MAO, AP 
Competing interest statement: The authors declare no potential conflict of interest.

Consent for publication: All the authors have given their consent for publication

Details of funding: none

Ethical Approval and Consent to participate: This study was approved by the Institutional Review Board of HUPNVS, Paris 7 University, AP-HP ( $\left.n^{\circ} 1343579\right)$. All of the patients signed a written consent form.

Keywords: Wilson disease, medication adherence, chronic disease, Trientine salts, DPenicillamine, zinc salts, efficacy, safety

Availability of data and materials: Please contact author for data requests.

Acknowledgements: none 


\section{Abstract}

Background: Wilson disease (WD) is one of the few genetic disorders that can be successfully treated with pharmacological agents. Copper-chelating agents (D-penicillamine and Trientine salts) and zinc salts have been demonstrated to be effective. There are two salts of trientine. Trientine dihydrochloride salt (TETA $2 \mathrm{HCL}$ ) is unstable at room temperature and requires storage at $2-8^{\circ} \mathrm{C}$. Trientine tetrahydrochloride (TETA $4 \mathrm{HCL}$ ) is a more stable salt of trientine that can be stored at room temperature. No comparative study between both salts of trientine has been performed to date. As the two chemical forms were available in France between 1970 and 2009 , we conducted a study to evaluate their efficacy and safety profiles.

Methods: This retrospective cohort study was conducted by reviewing data from the national WD registry in France. Forty-three WD patients who received TETA 2HCL or TETA 4HCL monotherapy for at least one year until 2010 were included. Primary endpoints were hepatic and neurological outcomes. Secondary endpoints were the events leading to a discontinuation of medication.

Results: Changes in medication were common, leading to the analysis of 57 treatment sequences of TETA 4HCL or TETA $2 \mathrm{HCL}$. The mean duration of treatment sequence was significantly longer in the TETA $4 \mathrm{HCL}$ group (12.6 years) than in the TETA 2HCL group (7.6 years) $(p=0.011)$. No difference in efficacy was detected. Both treatments were well tolerated except for a case of recurrence of lupus erythematosus-like syndrome in the TETA $2 \mathrm{HCL}$ group. The major reason for interruption of TETA $4 \mathrm{HCL}$ was due to a discontinuation in production of this salt. The reasons for stopping TETA $2 \mathrm{HCL}$ were mainly due to adherence issues largely attributed to the cold storage requirement.

Conclusions: The two salts of trientine were effective in treating patients with WD. However, interruption of TETA $2 \mathrm{HCL}$ was frequent, linked to the cold storage requirement. As adherence to treatment is a key factor in the successful management of WD, physicians need to be even 
more vigilant in detecting adherence difficulties in patients receiving treatment with TETA $2 \mathrm{HCL}$. 


\section{Introduction}

Wilson's disease (WD) is an autosomal recessive disorder characterized by pathological copper accumulation in many organs, initially the liver, and then essentially the cornea and brain. It is caused by homozygous or compound heterozygous mutations in the ATP7B gene which encodes a transmembrane copper-transporting P-type ATPase ${ }^{[1]}$. WD is one of the few genetic disorders that can be successfully treated with pharmacological agents. Treatment is based on the generation of a negative copper balance. Copper-chelating agents and zinc salts have been demonstrated to be effective in the treatment of WD, associated with a low copper $\operatorname{diet}^{[2]}$. However, the best therapeutic approach remains controversial because no randomized controlled trials have compared these treatments and the use of drugs depends mainly on center experience and access to treatment in different countries or regions. Treatment is a lifelong necessity and should be started as early as possible. Whatever the chosen medical therapy, non-adherence to or discontinuation of therapy is associated with a high risk of very severe hepatic or neurologic deterioration ${ }^{[3]}$. The optimum goal for patients requiring this lifelong medical therapy should therefore be to limit side effects and difficulties associated with treatment dispensation and conservation.

Copper-chelating agents (D-penicillamine and Trientine salt) bind with excess copper, forming a stable complex which is excreted mainly in the urine. It has been suggested that trientine may also decrease intestinal copper absorption ${ }^{[2,4]}$. Zinc salts decrease intestinal absorption of copper, inducing the synthesis of metallothioneins, proteins that sequester copper in the enterocytes ${ }^{[5]}$. Zinc salts are indicated in presymptomatic patients and during the maintenance phase of treatment ${ }^{[2]}$, but some data indicate that zinc may be considered also in patients who exhibit neurological symptoms during the acute phase of the disease ${ }^{[6]}$. Zinc is generally welltolerated in adults although gastritis and nausea may lead to discontinuation of treatment ${ }^{[7]}$. In presymptomatic children, gastrointestinal adverse effects are present in nearly $20 \%$ of the patients, associated with poor efficacy ${ }^{[8]}$. 
D-penicillamine is the reference treatment in many European countries, but severe adverse effects are frequent, leading to discontinuation of this therapy in up to $30 \%$ of patients ${ }^{[9]}$. The other copper chelators are trientine salts, currently indicated in WD patients who are intolerant to D-penicillamine. There are two currently available trientine salts. The trientine dihydrochloride salt (TETA $2 \mathrm{HCL}$ ) is unstable at room temperature and requires storage between 2 and $8^{\circ} \mathrm{C}$. In Europe, TETA $2 \mathrm{HCL}$ was approved by the Medicines Health and Regulatory Agency (MHRA) in the United Kingdom (UK) for the treatment of WD in 1985, and was supplied to some other European Union (EU) countries. In France, TETA $2 \mathrm{HCL}$ (Trientine ${ }^{\circledR}$ from Univar) was used through a compassionate use program for patients intolerant to D-penicillamine and was only dispensed by hospital pharmacies. This salt is now marketed in the EU as Cufence ${ }^{\circledR}$ following EU marketing authorization in 2019. Trientine tetrahydrochloride (TETA $4 \mathrm{HCL}$ ) is a more stable salt of trientine that can be stored at room temperature. In France, TETA 4HCL was available from the mid-1970s until 2009, as a hospital preparation supplied by AGEPS (Agence Générale des Equipements des Produits de Santé) of the Assistance Publique - Hôpitaux de Paris. This salt was granted a European marketing authorization in 2018 and is currently marketed in Europe as Cuprior ${ }^{\circledR}$ (Orphalan).

No comparative study between both salts of trientine (dihydrochloride and tetrahydrochloride) has been performed previously. As the two chemical forms were available in France between 1970 and 2009, we conducted a study to evaluate the efficacy and safety profiles of both salts.

\section{Materials and methods}

\section{Ethics approval and consent to participate}

All of the patients signed a written consent form. This study was approved by the Institutional Review Board of HUPNVS, Paris 7 University, AP-HP ( $\left.n^{\circ} 1343579\right)$.

\section{Patients}


This retrospective cohort study was conducted by reviewing data from the national WD registry. WD patients followed in Lariboisière hospital - Paris (National Centre for Wilson disease) and treated until 2010 were selected. This final date corresponded to the year after which the production of TETA 4HCL by AGEPS was discontinued. Diagnosis of WD was based on clinical symptoms, abnormal copper metabolism and genetic testing with a Leipzig score $\geq 4^{[2]}$. Only patients receiving TETA $2 \mathrm{HCL}$ or TETA $4 \mathrm{HCL}$ monotherapy for at least one year were included. The specific duration of one year of trientine monotherapy was considered as the minimum time required to show a treatment effect. Patients were in the initial or maintenance phase of treatment and received trientine as a first, second or third line treatment. Patients who received both forms of trientine (TETA 4HCL and $2 \mathrm{HCL}$ ) successively were included in the analysis. Patients who received either TETA $2 \mathrm{HCL}$ or TETA 4HCL in association with zinc salts were excluded.

\section{Analysis of treatments}

Different courses of treatment with TETA $4 \mathrm{HCL}$ and $2 \mathrm{HCL}$ were identified and treatment sequences of TETA 4HCL or $2 \mathrm{HCL}$ with a follow-up period superior to one year of continuous treatment were analyzed. Events leading to a discontinuation of medication were recorded and classified.

\section{Baseline comparison of treatments}

Clinical and laboratory data were recorded at the beginning and at the end of each trientine treatment sequence; the duration of the sequence was noted. The patients included in the study were divided into two groups according to the absence or presence of neurological symptoms. The hepatic assessment included clinical symptoms, measurement of serum transaminase levels, bilirubin and prothrombin time (PT). The presence of cirrhosis (typical findings on imaging and/or presence of clinical signs of portal hypertension) was recorded. Neurological evaluation was based on clinical symptoms. The presence of Kayser-Fleischer 
rings at slit-lamp examination was documented. Adherence to treatment was recorded by reports of compliance from the patients that were recorded in their medical records.

\section{Study endpoints}

Primary endpoints were hepatic and neurological outcomes. Hepatic outcome was based on clinical symptoms and course of liver enzymes and liver function tests. Neurological outcome was evaluated by neurological symptoms. Both hepatic and neurologic outcomes were scored as follows: unchanged, improved or deteriorated. The evolution of Kayser-Fleischer rings was also scored as unchanged, improved, disappeared or increased.

Secondary endpoints were the events leading to a discontinuation of medication. The reasons for treatment interruption or discontinuation were classified: loss of efficacy, adverse events, treatment non-adherence, manufacturing interruption.

\section{Statistical analyses}

Quantitative variables were expressed as median (interquartile range) and categorical variables as frequencies and percentages. Comparisons between two groups were made using the Student $U$ test for continuous variables and the Fisher exact test for qualitative variables if the frequency was $<5$, otherwise the chi-squared test was used.

\section{Results}

\section{Study group}

From the 248 WD patients recorded in the national registry and who were followed at the Lariboisière hospital before 2010, 62 received at least one sequence of treatment with a trientine salt. Nineteen patients were excluded because they received a zinc salt in combination with trientine over the treatment period. Thus, 43 patients were included in the study (Figure 1). Twenty-three were male (53.5\%) and the age at diagnosis was $21 \pm 9.3$ years (min 5.6; max 46.3). Nine patients (20.9\%) were diagnosed at the presymptomatic stage via familial screening, 19 presented with hepatic symptoms and 15 with neurological symptoms. 
Sixteen patients $(37 \%)$ had cirrhosis. Trientine was the first-line treatment for 4 patients (9.52\%). Trientine was prescribed as a second-line treatment after D-penicillamine in 35 patients, zinc salts in 2 patients and D-penicillamine associated with zinc salts in 2 other patients. D-penicillamine was stopped due to WD aggravation (2 cases) or due to the occurrence of adverse events in the remaining patients. The most common adverse events were renal disorders, thrombocytopenia and neutropenia, skin rash, digestive disorders and, less frequently, arthralgia, myasthenia-like syndrome and lupus-like syndrome. Zinc salts were stopped due to gastric irritation and in one case due to an increase in liver enzymes.

\section{Treatment sequences}

Changes in trientine treatment were common in this cohort. The 43 patients received 57 trientine monotherapy treatment sequences. This included 10 patients who received both TETA 4HCL and TETA $2 \mathrm{HCL}$ in different sequences (with a duration of more than one year); 2 patients who received only TETA $4 \mathrm{HCl}$ and 31 patients who received only TETA $2 \mathrm{HCL}$. This corresponded to 57 trientine treatment sequences: 13 sequences with TETA 4HCL and 44 sequences with TETA $2 \mathrm{HCL}$ (Figure 1).

\section{Baseline characteristics of TETA treatment sequences}

The mean sequence duration was significantly longer in the TETA 4HCL group, $151.7 \pm 111.1$ months vs $91.1 \pm 58.1$ months in the TETA $2 \mathrm{HCL}$ group $(p=0.011)$.

Table 1 presents a comparison of baseline parameters at the initiation of the treatment sequence in the two groups, TETA $2 \mathrm{HCL}$ and TETA 4HCL. Laboratory analyses were available only for a subset of patients due to the retrospective nature of the study. However, there were no statistically significant differences between groups relating to sex, age, laboratory values, and delay in onset of treatment. Regarding the initial phenotype, more patients in the TETA $4 \mathrm{HCL}$ group had neurological signs: $62 \%$ versus $43 \%$ in the TETA $2 \mathrm{HCL}$ group.

\section{Patient Outcomes}


The analysis of the evolution of hepatic and neurologic outcomes shows that the majority of the patients either improved clinically or their symptoms stabilized under the TETA treatment sequences (Table 2). Parameters relative to hepatic function, in particular serum transaminases, tended to improve (13 $(29.55 \%)$ in TETA $2 \mathrm{HCL}$ group versus $3(23.08 \%)$ in TETA 4HCL group), with no statistically significant differences observed between the two groups. Nevertheless, 3 patients worsened in the TETA $2 \mathrm{HCL}$ group.

Table 3 details the evolution between the two subgroups based on the presence of neurological symptoms at the sequence initiation. When hepatic symptoms were isolated at the initiation of the trientine sequence, no neurological symptoms developed. When only neurological symptoms were evident at the initiation of the treatment sequence, they improved or remained unchanged for all except for one TETA $2 \mathrm{HCL}$ sequence.

Kayser-Fleischer (KF) ring evolution was comparable in both treatment groups. In one sequence of the TETA 4HCL group, a slight increase in the ring was reported, without deterioration in neurological and hepatic disease (Table 4).

\section{Reasons for discontinuation of trientine treatment and adverse effects}

No adverse effects were observed during the TETA 4HCL sequences (mean duration: 12.6 years). All 13 TETA 4HCL sequences were stopped during the study period: 11 (85\%) due to the fact that manufacturing of the hospital preparation was discontinued, 1 due to difficulties with supply and 1 due to an increase in Kayser-Fleischer ring, without neurologic or hepatic deterioration.

The mean duration of treatment sequence was 7.6 years for TETA 2HCL. At the end of the study period, $26(60 \%)$ of TETA $2 \mathrm{HCL}$ monotherapy treatments were still ongoing. In three patients, zinc was added to TETA $2 \mathrm{HCL}$ due to hepatic or neurological deterioration in two cases and as a result of an increase in liver copper without hepatic deterioration in one case. A bad adherence to TETA $2 \mathrm{HCL}$ was suspected in these three cases. The reasons for stopping 
TETA $2 \mathrm{HCL}$ were mainly due to adherence to medication issues (11 cases), generally linked to the requirement for cold storage. Other reasons included one case of lupus erythematosuslike syndrome in a patient with a previous diagnosis of lupus erythematosus during treatment with D-penicillamine, and one liver transplantation for suspicion of hepatocellular carcinoma. Two patients died (salivary gland neoplasm and suicide); these deaths were not considered as related to WD.

\section{Discussion}

Now that TETA 4HCL has received a European marketing authorization and is being marketed in Europe (as Cuprior ${ }^{\circledR}$ ), this study comparing the efficacy and safety of TETA 4HCL to TETA $2 \mathrm{HCL}$ in everyday clinical practice is important since many patients are still taking TETA $2 \mathrm{HCL}$. Efficacy and safety are closely interrelated because any switch in treatment is usually linked to a lack of efficacy, observance issues, adverse effects or difficulties with treatment adherence.

In France, between 1970 and 2009, both trientine salts were available. All patients with WD in France are included in a national registry, allowing the possibility to conduct this retrospective study. Trientine monotherapy for more than 12 months was evaluated in 43 patients out of the 248 patients included in the WD registry in 2010, which represents a large cohort in this rare disease.

In accordance with French guidelines, trientine was mainly prescribed as a second-line treatment after D-penicillamine (81\% of the patients). This switch was mainly due to the occurrence of adverse events. Adverse drug reactions are commonly reported with Dpenicillamine treatment and are serious enough to lead to at least $30 \%$ of patients on Dpenicillamine discontinuing the drug ${ }^{[10]}$. Four patients received zinc salts as first-line treatment, associated or not with D-penicillamine. Discontinuation of zinc therapy due to adverse effects such as gastrointestinal symptoms, is common in patients with WD in children as in adults ${ }^{[7,8]}$. 
The study population is representative of the wide WD population, as it includes children and adult patients. Mean age at diagnosis was 20 years (range 5.6 years to 46.3 years). The majority of patients presented with liver disease. These data are comparable to those of former studies $^{[7,9]}$. Although there was non-random allocation to treatment group, the baseline characteristics of patients at the beginning of the sequences were relatively balanced.

The majority of patients either improved or stabilized their symptoms under trientine. No differences in efficacy were detected when assessing TETA 4HCL and $2 \mathrm{HCL}$ treatment sequences for changes in hepatic and neurological symptoms. Parameters relative to hepatic function, in particular serum transaminase levels, tended to improve in both groups. Many studies have demonstrated the effectiveness of TETA $2 \mathrm{HCL}$, showing in addition that fewer side effects are observed than with D-penicillamine ${ }^{[9,11]}$. In this study, three patients on TETA 2HCL had hepatic or neurological deterioration and a bad adherence to treatment was highly suspected in these cases. In WD, during long-term follow-up, the most important cause of hepatic and/or neurologic worsening, leading sometimes to death, is non-adherence to WD treatment ${ }^{[12,13,14,15]}$. Up to $50 \%$ of patients are non-compliant with treatment ${ }^{[16,17]}$. The identification of factors which compromise adherence to medication remains difficult and findings are often contradictory. However, it is evident that barriers to treatment dispensation or conservation should be minimized. TETA $2 \mathrm{HCL}$ requires refrigerated storage between 2 and $8^{\circ} \mathrm{C}$. This is certainly an important disadvantage for patients who need to take the drug several times throughout the day, while studying, working or travelling, on a daily basis for the duration of their lives.

In this study, the main reason for discontinuation of the drug was the production shutdown of one of the biochemical salt, TETA 4HCL. All 13 TETA 4HCL treatment were stopped due to cessation of manufacturing or supply difficulties, except for 1 patient whose Kayser-Fleischer rings increased. No adverse events were reported in this group following average treatment duration of 12.5 years. In the TETA $2 \mathrm{HCL}$ group $(n=44)$, only one adverse event was reported. This was a case of lupus erythematosus-like syndrome in a patient who already had presented 
with penicillamine-induced lupus erythematosus-like syndrome. However, 11 treatment sequences were stopped as a result of difficulties in treatment adherence due to the refrigeration requirements (often creating problems for patients who needed to work away from home or travel) for TETA 2HCL. Published guidelines indicate that adverse effects are rarely observed with TETA $2 \mathrm{HCL}$ treatment: urticaria, reversible anemia and lupus-like reactions are described as the key potential side effects ${ }^{[2,18]}$. In our study, TETA 4HCL was also shown to be well tolerated, consistent with the review of Allery that describes a TETA 4HCL safety profile comparable to that of TETA $2 \mathrm{HCL}^{[19]}$.

The number of patients treated with trientine as a first-line therapy is small and does not allow for a satisfactory analysis of this sub-group. However, 6 other patients started trientine therapy early in the course of this lifelong disease, during the first three months following diagnosis. Trientine was introduced in these patients as a result of early adverse events associated with D-penicillamine. The clinical evolution of these patients was not different to that of the whole cohort.

This study has certain limitations, including its retrospective nature and the lack of randomization. The rarity of the disease, the fact that trientine was used in France via a compassionate use program and that manufacturing of TETA 4HCL was discontinued in 2009, explain the low number of patients included in the study. At that time, adherence was subjectively assessed during the medical examination and not with dedicated scores such as the Morisky score ${ }^{[20]}$. However, it was possible to analyze data quite exhaustively over long periods of treatment under trientine (7.6 years for TETA $2 \mathrm{HCL}$ and 12.6 years for TETA 4HCL).

In conclusion, both trientine salts were equally effective in controlling WD. Adverse events were infrequent. In WD, adherence to medication is a key factor for treatment success. Interruption of TETA $2 \mathrm{HCL}$ therapy was frequent, linked to the requirement for cold storage. Physicians therefore have to be even more vigilant to detect non-adherence to medication as early as possible in patients being treated with TETA $2 \mathrm{HCL}$. 


\section{Legends Tables}

Table 1 Baseline characteristics at TETA sequence initiation

Table 2 Hepatic and neurologic evolution in all patients

Table 3 Outcome in patients based on presence of neurological symptoms at the sequence initiation

Table 4 Evolution of the Kayser-Fleischer ring between sequence initiation and sequence end.

\section{Legends Figure}

Figure 1 Flow-chart of patients included and treatment sequences 


\section{References}

1. Poujois A, Woimant F. Wilson's disease: A 2017 update. Clin Res Hepatol Gastroenterol. 2018 Dec; 42(6):512-520.

2. EASL Clinical Practice Guidelines: Wilson's disease. European Association for Study of Liver. J Hepatol. 2012 Mar; 56(3):671-85.

3. Masełbas W, Członkowska A, Litwin T, Niewada M. Persistence with treatment for Wilson disease: a retrospective study. BMC Neurol. 2019 Nov 12; 19(1):278.

4. Siegemund R, Lößner J, Günther K, Kühn HJ, Bachmann H. Mode of action of triethylenetetramine dihydrochloride on copper metabolism in Wilson's disease. Acta Neurol Scand. 1991 Jun; 83(6):364-6.

5. Brewer GJ. Zinc acetate for the treatment of Wilson's disease. Expert Opin Pharmacother. 2001 Sep; 2(9):1473-7.

6. Członkowska A, Litwin T, Karlinski M et al. D-penicillamine versus zinc sulfate s first-line therapy for Wilson's disease. Eur J Neurol 2014 ; 21: 599-606.

7. Weiss KH, Gotthardt DN, Klemm D, Merle U, Ferenci Foerster D, Schaefer M, et al. Zinc monotherapy is not as effective as chelating agents in treatment of Wilson disease. Gastroenterology. 2011 Apr; 140(4):1189-1198.e1.

8. Santiago R, Gottrand F, Debray D, Bridoux L, Lachaux A, Morali A, et al. Zinc Therapy for Wilson Disease in Children in French Pediatric Centers. J Pediatr Gastroenterol Nutr. 2015 Dec; 61(6):613-8.

9. Weiss KH, Thurik F, Gotthardt DN, Schäfer M, Teufel U, Wiegand F, et al. Efficacy and safety of oral chelators in treatment of patients with Wilson disease. Clin Gastroenterol Hepatol. 2013 Aug; 11(8):1028-35.e1-2.

10. Litwin T, Członkowska A and Socha P. Oral Chelator Treatment of Wilson Disease: DPenicillamine. In clinical and translational perspectives on Wilson Disease. Edited by Kerkar $\mathrm{N}$ and Roberts EA, Elsevier 2019. p 357-363.

11. Merle U, Schaefer M, Ferenci P, Stremmel W. Clinical presentation, diagnosis and longterm outcome of Wilson's disease: a cohort study. Gut. $2007 ; 56: 115-20$.

12. Walshe JM, Dixon AK. Dangers of non-compliance in Wilson's disease. Lancet. 1986; 1:845-7.

13. Maselbas W, Chabik G, Czlonkowska A. Persistence with treatment in patients with Wilson disease. Neurol Neurochir Pol. 2010; 44:260-63

14. Weiss $\mathrm{KH}$, Stremmel W. Clinical considerations for an effective medical therapy in Wilson's disease. Ann N Y Acad Sci. 2014; 1315:81-5 
15. Dziezyc K, Karlinski M, Litwin T, Członkowska A. Compliant treatment with anti-copper agents prevents clinically overt Wilson's disease in pre-symptomatic patients. Eur $\mathrm{J}$ Neurol. 2014; 21:332-37.

16. Maselbas W, Litwin T, Czlonkowska A. Social and demographic characteristics of a Polish cohort with Wilson disease and the impact of treatment persistence. Orphanet Journal of Rare Disease 2019; 14:167; doi: 10.1186/s13023-019-1133-28

17. Jacquelet E, Beretti J, De-Tassigny A, Girardot-Tinant N, Wenisch E, Lachaux A, Pheulpin MC, Poujois A, Woimant F. Compliance with treatment in Wilson's disease: on the interest of a multidisciplinary closer follow-up. Rev Med Interne. 2018; 39:155-60.

18. Socha P, Janczyk W, Dhawan A, Baumann U, D'Antiga L, Tanner S, lorio R, Vajro P, Houwen R, Fischler B, Dezsofi A, Hadzic N, Hierro L, Jahnel J, McLin V, Nobili V, Smets F, Verkade HJ, Debray D. Wilson's Disease in Children: A Position Paper by the Hepatology Committee of the European Society for Paediatric Gastroenterology,Hepatology and Nutrition.J Pediatr Gastroenterol Nutr. 2018; 66:33444.

19. Allery C. Maladie de Wilson. Place de la Trientine dans la stratégie thérapeutique. Thèse de doctorat en pharmacie. Université Paris Descartes 2013.

20. Morisky DE, Green LW, Levine DM. Concurrent and predictive validity of a self-reported measure of medication adherence. Med Care. 1986 Jan;24(1):67-74. doi: 10.1097/00005650-198601000-00007. 

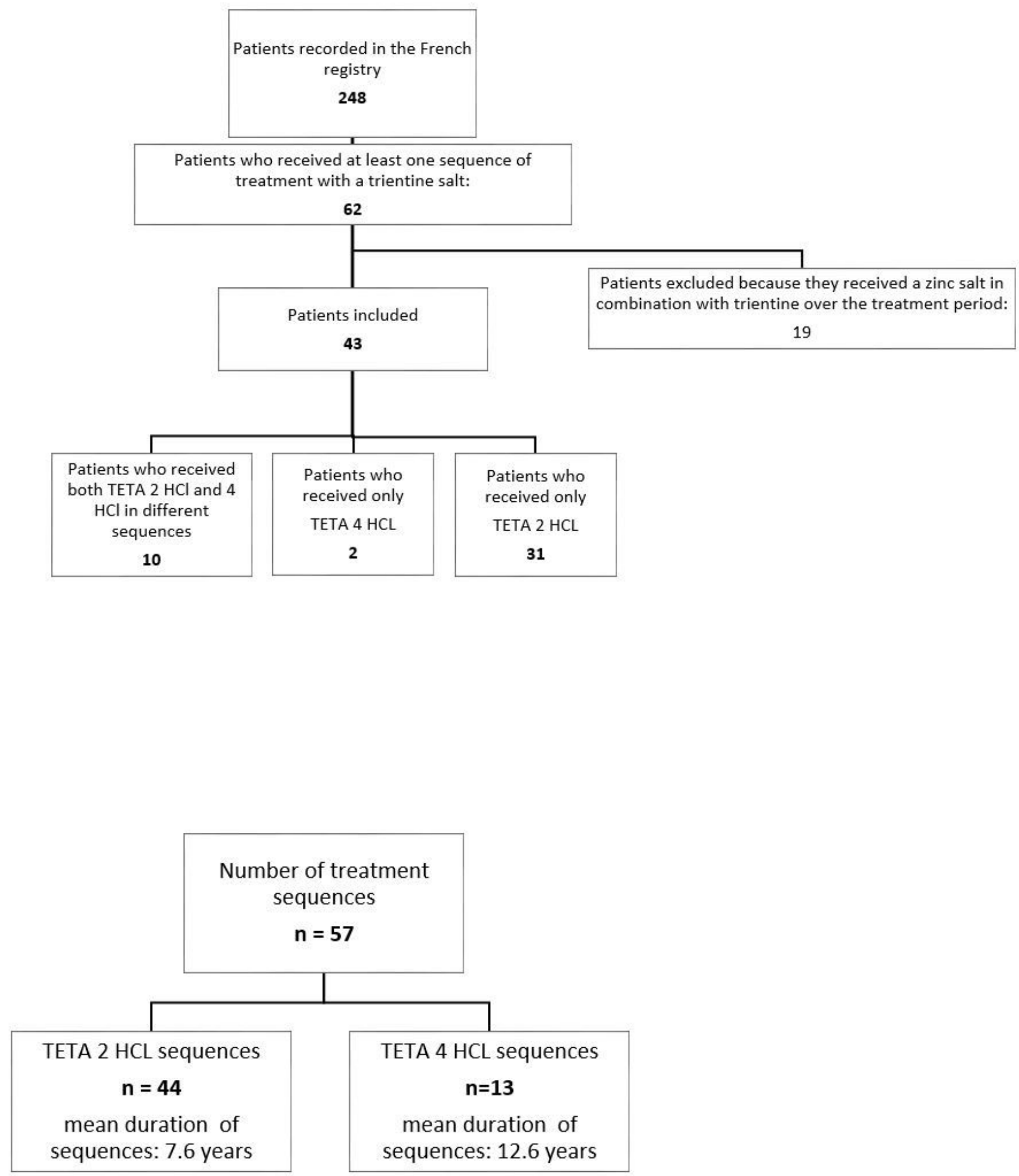

\section{Figure 1}

Flow-chart of patients included and treatment sequences. 